\title{
Muonic-atom scattering from hydrogenic liquids in an incoherent approach
}

\author{
Andrzej Adamczak a and Andrzej Z. Górski \\ Institute of Nuclear Physics Polish Academy of Sciences, Radzikowskiego 152, 31342 Kraków, Poland
}

Received 27 November 2017 / Received in final form 23 December 2017

Published online 8 March 2018

(C) The Author(s) 2018. This article is published with open access at Springerlink.com

\begin{abstract}
The differential cross sections for low-energy muonic hydrogen atom scattering in liquid hydrogenic targets have been calculated in the incoherent approximation using the Van Hove response function. A simple model of liquids and the available experimental parameters have been employed for a description of the diffusive and vibrational modes in these targets. At collision energies below about $10 \mathrm{meV}$, the obtained cross sections are very different from the analogous cross sections for scattering in hydrogenic gases.
\end{abstract}

\section{Introduction}

Many experiments in low-energy muon physics were performed in the liquid mixtures of hydrogen isotopes. In particular, such hydrogenic targets were employed for studying the muon-catalyzed $p d$ and $p t$ fusion in the muonic molecules $p d \mu$ and $p t \mu$ (see, e.g., [1-5]). The yields of different products from these fusion reactions strongly depend on the populations of hyperfine states of the muonic molecules. These yields are functions of the concentrations of deuterium and tritium admixtures in the $\mathrm{H}_{2}$ targets, which is known as the Wolfenstein-Gershtein effect $[6,7]$. This is due to a complicated chain of processes from the Coulomb capture of the negative muons to formation of the muonic molecules. The time evolution of the spin and kinetic energy of the $d \mu$ and $t \mu$ atoms is of particular importance. In order to compare the theoretical fusion rates with the experimental data, it is necessary to accurately describe the kinetics of muon-catalyzed fusion. Detailed kinetics calculations [3-5], were performed using then available cross sections for scattering of the muonic atoms from the hydrogen-isotope nuclei [8-10]. However, in the considered experiments, these processes take place in the molecular liquid targets. One may thus expect that the realistic cross sections are very different from that for the scattering from free nuclei or molecules, which has already been shown in the case of solid hydrogenic targets [11]. An estimation of the differential cross sections for muonic atom scattering from the liquid hydrogenic targets in a simple incoherent approximation is the aim of this work. The obtained results can be applied for an accurate simulation of the new experimental study of muon-catalyzed $p t$ fusion, which is underway at JINR in
Dubna. This experiment has been planned in order to clarify the large discrepancies between theory and experiment in the $p t \mu$ case, and to observe more fusion channels (e.g., pt $\mu \rightarrow{ }^{4} \mathrm{He}+e^{+}+e^{-}$) for the first time [12,13].

A method of estimating the differential cross sections for muonic hydrogen atom scattering in liquid hydrogenic targets in the incoherent approximation is presented in Section 2. Some examples of the calculated cross sections for the liquid hydrogen, deuterium, and hydrogen with a small admixture of deuterium or tritium, at the temperature $T=22 \mathrm{~K}$ and saturated-vapor pressure, are shown in Section 3. A summary of the obtained results and conclusions are given in Section 4.

\section{Method of calculation}

Since a muonic hydrogen atom $a \mu(a=p, d$, or $t)$ is a small neutral object, the cross section for $a \mu$ scattering in a condensed target can be calculated using the methods that were developed for a description of the neutron scattering. In the incoherent approximation, the partial differential cross section per a single target molecule can be expressed in terms of the incoherent fraction $\mathcal{S}_{i}(\boldsymbol{\kappa}, \omega)$ of the Van Hove response function $\mathcal{S}(\boldsymbol{\kappa}, \omega)$ [14]

$$
\frac{\partial^{2} \sigma}{\partial \Omega \partial \varepsilon^{\prime}}=\frac{k^{\prime}}{k} \sigma_{\mathrm{mol}} \mathcal{S}_{i}(\vec{\kappa}, \omega) \text {. }
$$

The squared amplitude for $a \mu$ scattering from a free hydrogenic molecule is denoted here by $\sigma_{\text {mol }}$, the energy transfer $\omega$ and momentum transfer $\boldsymbol{\kappa}$ to the condensed target are defined as

$$
\omega=\varepsilon-\varepsilon^{\prime}-\Delta \mathrm{E}, \quad \boldsymbol{\kappa}=\boldsymbol{k}-\boldsymbol{k}^{\prime}
$$

\footnotetext{
${ }^{\mathrm{a}}$ e-mail: andrzej.adamczak@ifj.edu.pl
} 
where $\varepsilon$ and $\varepsilon^{\prime}$ are the initial and final kinetic energies of the atom; vectors $\boldsymbol{k}$ and $\boldsymbol{k}^{\prime}$ denote the corresponding momenta. The energy transfer due to internal transitions in the atom and the target molecule is denoted by $\Delta \mathrm{E}$. equation (1) is exact for the incoherent processes of internal transitions in the atom (spin flip) or molecule (rotational-vibrational excitations). In the case of strictly elastic scattering, the approximation $\mathcal{S} \approx \mathcal{S}_{i}$ is valid for higher momentum transfers, when the coherent processes are relatively small. It has been shown in reference [11] that the total cross sections for $a \mu$ scattering in solid hydrogens are practically unaffected by the coherent processes at collision energy $\varepsilon \gtrsim 1-2 \mathrm{meV}$. In equation (1), the rotational-vibrational transitions in the target molecules and the spin-flip transitions in the scattered atom $a \mu$ are taken into account in the free-molecule squared amplitudes $\sigma_{\text {mol }}$, which were calculated in reference [15] for all combinations of the hydrogen isotopes. These calculations employed the amplitudes for $a \mu$ scattering from the bare hydrogen-isotope nuclei $[9,10]$ as the input.

Function $\mathcal{S}_{i}$ in equation (1) describes the translative motion of molecules in a liquid target. The studies of liquid $\mathrm{H}_{2}$ and $\mathrm{D}_{2}$ using slow neutron scattering showed that both the diffusive and collective modes are present in the dynamics of these quantum liquids (see, e.g., Refs. [16-19] and references therein). These studies revealed the phonon spectra similar to those characteristic for polycrystalline powders. Thus, despite the lack of a periodic structure in the liquid hydrogens, it is reasonable to describe their properties in terms of the Debye temperature for a certain range of the momentum transfers. At lowest energies, the scattering in liquids is dominated by the diffusive motion of target particles, which results in the presence of a broad quasielastic peak centered at the incident energy. At the liquid hydrogen density, the interactions between the neighboring molecules are important. This leads to a recoil-less scattering at lowest $\kappa$ and $\omega$. For example, such effect was observed in the lowest rotational excitation of $\mathrm{H}_{2}$ in collision with neutrons [20].

Many advanced theoretical models and computer programs were developed for a description of neutron scattering from liquid $\mathrm{H}_{2}$ and $\mathrm{D}_{2}$ (see, e.g., Refs. [21-23]). These models lead to different forms of the response function, which is usually convoluted with a response from the integral degrees of freedom of the molecules $\mathrm{H}_{2}$ and $\mathrm{D}_{2}$. Such convolution involves the spin correlations that are characteristic for the neutron scattering from these molecules and different from the analogous case of muonic hydrogen atom scattering. In our approach, the spin correlations and the rotational-vibrational structure of the molecules are already taken into account in the squared amplitudes $\sigma_{\text {mol }}$. For these reasons, below we evaluate the response function for the specific case of muonic atom scattering, using a simple general model from reference [24].

In liquid hydrogens, the diffusive motion at small $\kappa$ is well described [16] by the Langevin equation

$$
M \frac{\mathrm{d}^{2} \boldsymbol{R}}{\mathrm{d} t^{2}}=-\zeta M \frac{\mathrm{d} \boldsymbol{R}}{\mathrm{d} t}+\boldsymbol{F}_{s}(t)
$$

where $M$ is the molecular mass, $\boldsymbol{R}$ is a position of the molecule, $\zeta$ denotes the strength of a frictional force due to the movement in liquid, and $\boldsymbol{F}_{s}$ is a stochastic force connected with the scattering from other molecules. The coefficient $\zeta$ is related to the self-diffusion coefficient $D_{s}$ by the Einstein relation

$$
\zeta=k_{B} T /\left(M D_{S}\right)
$$

in which $k_{B}$ denotes Boltzmann's constant. Equations (3) and (4) lead to the following diffusion contribution [24]:

$$
\begin{aligned}
\mathcal{S}_{\text {diff }}(\kappa, \omega)= & \frac{1}{\pi} \exp (-2 W) \beta_{T} \omega\left[n_{B}(\omega)+1\right] \\
& \times \frac{D_{s} \kappa^{2}}{\omega^{2}+\left(D_{s} \kappa^{2}\right)^{2}},
\end{aligned}
$$

to the response function $\mathcal{S}_{i}$. Function $\exp (-2 W)$ denotes the Debye-Waller factor and the Bose factor $n_{B}(\omega)$ is defined as

$$
n_{B}=\left[\exp \left(\beta_{T} \omega\right)-1\right]^{-1},
$$

where $\beta_{T}=1 /\left(k_{B} T\right)$. Equation (5) can be applied for small $\kappa$ and $\omega$, apart from the limit $\kappa, \omega \rightarrow 0$. The width $\Delta_{\text {diff }}$ at the half maximum of the quasi-elastic Lorentzian factor in equation (5) equals $2 D_{s} \kappa^{2}$. Since the neutron experiments revealed that $\Delta_{\text {diff }}$ is almost constant at higher momentum transfers, we fix its value at $\kappa>\kappa_{\max }$. For example, $\kappa_{\max } \approx 1.7 \AA^{-1}$ in the case of $20-\mathrm{K}$ liquid deuterium [16].

The collective-motion contribution $\mathcal{S}_{\text {pho }}$ to $\mathcal{S}_{i}$ can be described using the following incoherent phonon expansion for a harmonic crystal $[14,24]$ :

$$
\mathcal{S}_{\mathrm{vib}}(\kappa, \omega)=\exp (-2 W) \sum_{n=1}^{\infty} g_{n}(\omega)(2 W)^{n} / n !
$$

without the strictly elastic term $\delta(\omega)$, which is characteristic for scattering in solids. Functions $g_{n}$, which describe the subsequent $n$-phonon processes, and the exponent $2 W\left(\kappa^{2}\right)$ of the Debye-Waller factor are given in reference [11] for the isotropic Debye model of a solid. We estimate the effective Debye temperature $\Theta_{D}$ using the relation:

$$
\Theta_{D}=\frac{h c_{s}}{k_{B}}\left(\frac{3}{4 \pi} \frac{N_{A}}{V}\right)^{2},
$$

where $h$ is Planck's constant, $c_{s}$ denotes the sound velocity, $N_{A}$ is Avogadro's constant, and $V$ represents the molar volume. The corresponding Debye energy is defined as $\omega_{D}=k_{B} \Theta_{D}$.

In theory of liquids, a generalized frequency spectrum $Z(\omega)$ was introduced [25], which is analogous to the density of vibrational states in solids. The Langevin equation (3) leads to the following contribution from the 


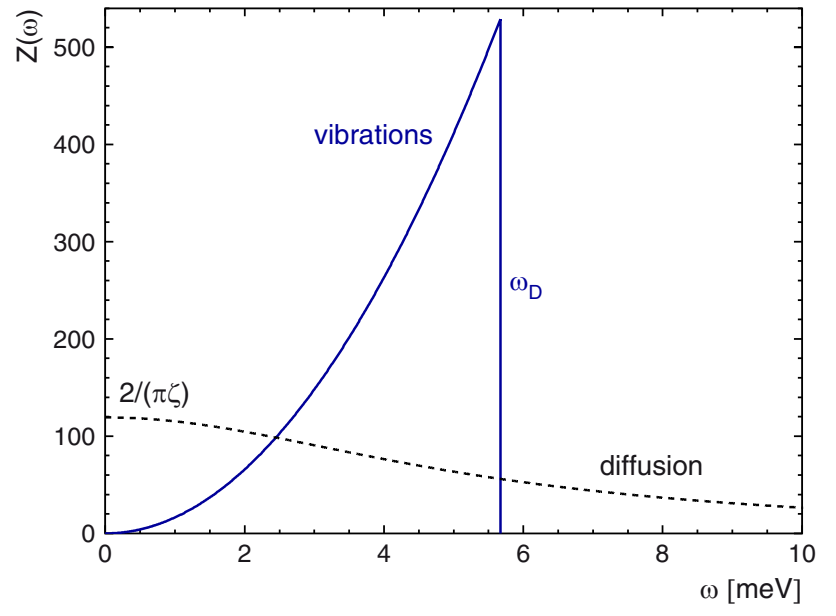

Fig. 1. Generalized frequency spectrum $Z(\omega)$ (in arbitrary units) for the simple model of $22-\mathrm{K}$ liquid hydrogen.

diffusive motion:

$$
Z_{\mathrm{diff}}(\omega)=\frac{2}{\pi \zeta} \frac{\zeta^{2}}{\omega^{2}+\zeta^{2}}
$$

to $Z(\omega)$. A contribution from the collective vibrations is given by the standard Debye density $Z_{\mathrm{vib}}(\omega)$ of vibrational states. These two contributions are shown in Figure 1 for the 22-K liquid hydrogen at saturated-vapor pressure. The values of parameters $D_{s}, c_{s}$, and $V$ are taken from reference [26]. The presented plot exhibits characteristic features, which are observed in liquids. The spectrum at lowest $\omega$ is determined by the diffusive modes. In particular, $Z(\omega)$ tends to a finite value when $\omega$ approaches zero. For solids, this limit equals zero. The Debye frequency $\Theta_{D}$ corresponds to the large peaks in liquids, which are due to the finite-frequency collective oscillations. The tail at large $\omega$, which is apparent in the experiments, is consistent the Enskog theory for a fluid of hard spheres [27].

For the first estimation of the cross sections for muonic atom scattering in liquid hydrogens, we use a sum of the functions (5) and (7)

$$
\mathcal{S}_{i}(\kappa, \omega) \approx \mathcal{S}_{\text {diff }}(\kappa, \omega)+\mathcal{S}_{\mathrm{vib}}(\kappa, \omega)
$$

as a fair approximation of the total response function. Figure 2 presents the function (10) for the $22-\mathrm{K}$ liquid $\mathrm{H}_{2}$ at saturated-vapor pressure. In this plot, one can clearly distinguish the diffusive contribution at the lowest $\omega$, and then the subsequent contributions from the one-phonon and two-phonon processes.

For large $\kappa$ and $\omega$, any system (apart from liquid helium at lowest temperatures) is accurately described by the asymptotic Gaussian form [14,24]

$$
\mathcal{S}_{i}(\kappa, \omega)=\exp \left[-\left(\omega-\omega_{R}\right)^{2} / \Delta_{R}^{2}\right], \quad \Delta_{R}=\sqrt{8 \mathcal{E}_{T} \omega_{R} / 3}
$$

in which $\omega_{R}=\kappa^{2} /(2 M)$ denotes the recoil energy and $\mathcal{E}_{T}$ is the mean kinetic energy of a hydrogenic molecule

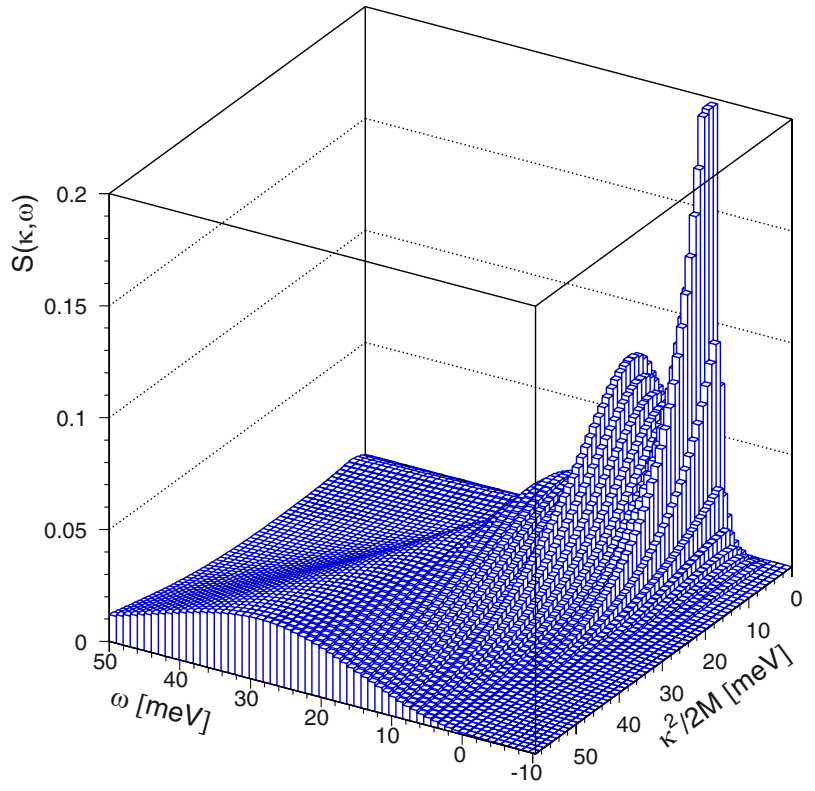

Fig. 2. Incoherent response function (10) for the $22-\mathrm{K}$ liquid hydrogen at saturated-vapor pressure.

in the considered liquid. In solid and liquid hydrogens, this energy is significantly greater than the Maxwellian energy $\frac{3}{2} k_{B} T$ owing to large zero-point vibrations of the light hydrogenic molecules in these quantum systems [28]. The mean kinetics energy can be estimated using the following formula

$$
\mathcal{E}_{T}=\frac{3}{2} \int_{0}^{\infty} \mathrm{d} \omega Z_{\mathrm{vib}}(\omega) \omega\left[n_{B}(\omega)+\frac{1}{2}\right] .
$$

The asymptotic response function (11) leads to a correct free-molecule limit at large energy transfers.

\section{Results of calculations}

Our calculations of the cross sections have been performed for liquid hydrogen and deuterium at $22 \mathrm{~K}$ and saturatedvapor pressures, which corresponds to the conditions of the experiments performed at PSI [3,4] and JINR [12]. At $22 \mathrm{~K}$, practically all symmetric molecules $\mathrm{H}_{2}$ and $\mathrm{D}_{2}$ are in the ground rotational state $K=0$. Thus, we are dealing with the liquid parahydrogen (para- $\left.\mathrm{H}_{2}\right)$ and orthodeuterium (ortho- $\mathrm{D}_{2}$ ). Only freshly prepared targets are statistical mixtures of the rotational states $K=0$ and $K=1$, due to a slow rotational deexcitation of the symmetric molecules [26]. Such targets are often called normal hydrogens and labeled as $n \mathrm{H}_{2}$ and $n \mathrm{D}_{2}$, respectively.

An estimation of the Debye temperature using equation (8) and the parameters $c_{s}$ and $V$ from reference [26] results in $\Theta_{D} \approx 66 \mathrm{~K}$ and $\omega_{D} \approx 5.7 \mathrm{meV}$, for both hydrogen and deuterium. For our conditions, the value of self-diffusion coefficient $D_{s}$ equals $1.12 \times 10^{-4} \mathrm{~cm}^{2} / \mathrm{s}$ for hydrogen and $0.49 \times 10^{-4} \mathrm{~cm}^{2} / \mathrm{s}$ for deuterium [26]. These parameters have been applied for plotting Figures 1 and 2, and for calculating the cross sections. In order to illustrate 


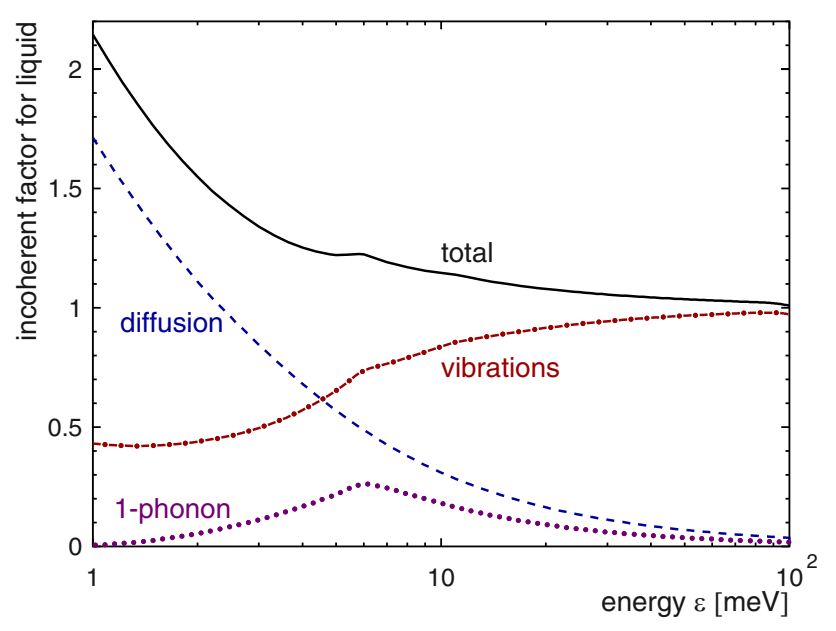

Fig. 3. Function $\mathcal{C}_{\text {inc }}$ for $p \mu$ scattering in the $22-\mathrm{K}$ liquid hydrogen. The contributions to $\mathcal{C}_{\text {inc }}$ from the diffusive and vibration modes are shown separately. The label "1-phonon" denotes the process of one-phonon creation.

contributions from the diffusive and vibration modes to the total cross section, the following function

$$
\mathcal{C}_{\text {inc }}(\varepsilon)=\frac{1}{4 \pi}\left(\frac{M_{a \mu}}{\mathcal{M}}\right)^{2} \int \mathrm{d} \Omega \mathrm{d} \varepsilon^{\prime} \frac{k^{\prime}}{k} \mathcal{S}_{i}(\kappa, \omega)
$$

is shown in Figure 3. In equation (13), $M_{a \mu}$ denotes the mass of $a \mu$ and $\mathcal{M}$ is a reduced mass of the system which consists of the impinging muonic atom and a single hydrogenic molecule. Function $\mathcal{C}_{\text {inc }}$ is a ratio of the cross section for $a \mu$ scattering from this molecule in the liquid target and the corresponding cross section for the same free molecule. In definition (13), it is assumed that the latter cross section is constant. Function $\mathcal{C}_{\text {inc }}$ falls from the maximal value of $\left(M_{a \mu} / \mathcal{M}\right)^{2}$, at $\varepsilon \rightarrow 0$, to the free-molecule limit of 1 at $\varepsilon \gtrsim 100 \mathrm{meV}$. The quasielastic diffusive mode is most important at lowest energies. The vibration mode includes annihilation and creation of one phonon and many phonons. The plotted one-phonon contribution to $\mathcal{C}_{\text {inc }}$ has a maximum at the Debye energy.

The total cross section for scattering of the $p \mu$ atom from liquid para- $\mathrm{H}_{2}$ is shown in Figure 4. The atom is in the ground spin state $F=0$ during the scattering process. The contributions to the cross section from the molecular motion in liquid para- $\mathrm{H}_{2}$ and from the rotational-vibrational excitations of the target molecule $\mathrm{H}_{2}$ are shown separately. The latter contribution begin to appear at $\varepsilon \approx 40 \mathrm{meV}$, which corresponds to the first rotational excitation $K=0 \rightarrow 2$ of $\mathrm{H}_{2}$. The transition $K=0 \rightarrow 1$ is forbidden for the state $F=0$ of $p \mu$. The doubled total cross section for $p \mu(F=0)$ scattering from a bare proton [9] is shown for a comparison. In this Figure, three regimes of scattering are visible. For $\varepsilon \lesssim 100 \mathrm{meV}$, effects of the target molecule interactions with the neighboring $\mathrm{H}_{2}$ molecules are important. Above this energy, the scattering passes to the free-molecule regime. Finally, above about $1 \mathrm{eV}$ (depending on the choice of hydrogen isotopes [15]), the free-nuclei regime is achieved.

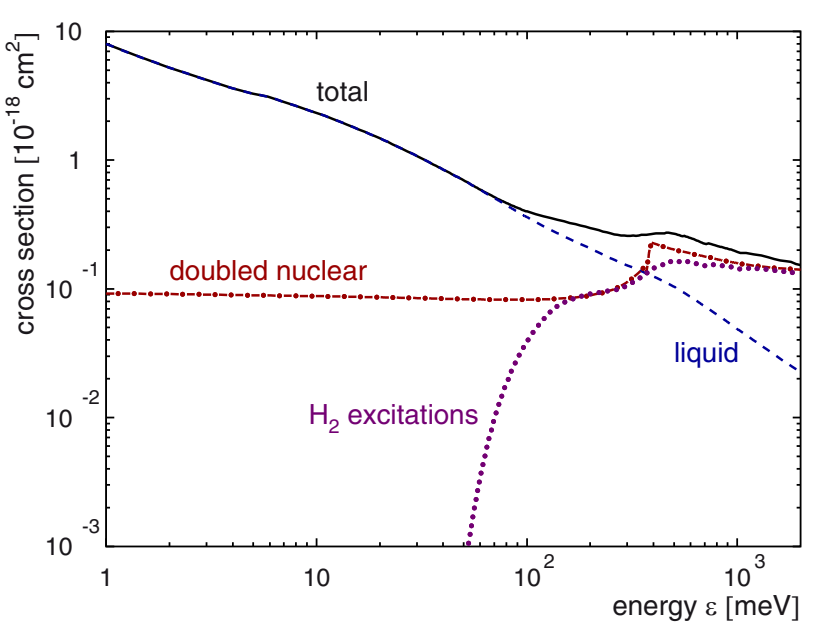

Fig. 4. Total cross section for $p \mu(F=0)$ scattering in the $22-\mathrm{K}$ liquid para- $\mathrm{H}_{2}$. The label "liquid" denotes scattering without internal excitations of the target molecules. The label " $\mathrm{H}_{2}$ excitations" represents a contribution from the rotationalvibrational excitations of the $\mathrm{H}_{2}$ molecule. The label "doubled nuclear" denotes the doubled cross section for the elastic scattering $p \mu(F=0)+p$.

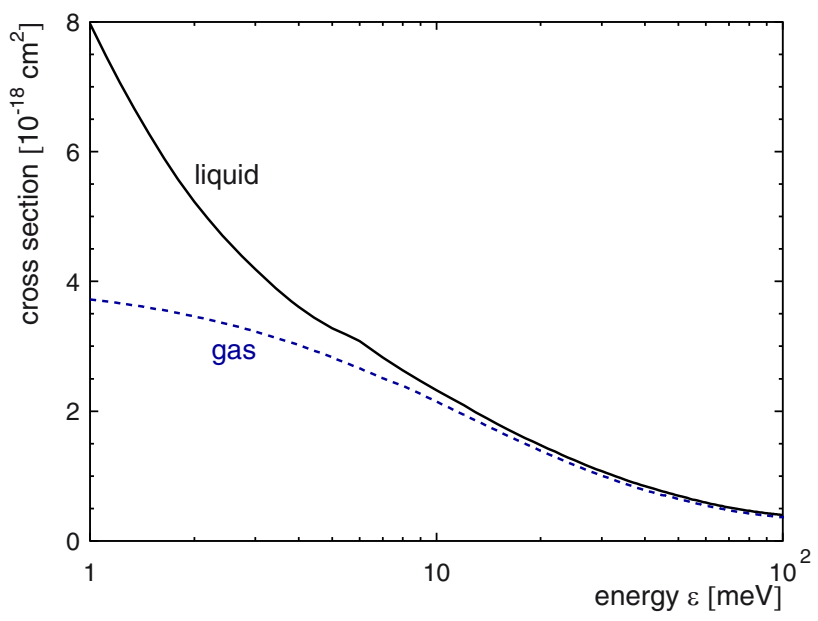

Fig. 5. Total cross sections for $p \mu(F=0)$ scattering in the 22-K liquid and gaseous para- $\mathrm{H}_{2}$.

The total cross sections for $p \mu(F=0)$ scattering in the liquid and gaseous parahydrogen are shown in Figure 5. One can see that the cross sections begin to significantly diverge below $10 \mathrm{meV}$. The differential cross sections for $a \mu$ scattering from the hydrogenic targets are strongly anisotropic. This can be conveniently shown using the transport cross sections

$$
\sigma_{\operatorname{tr}}(\varepsilon)=\int \mathrm{d} \Omega \mathrm{d} \varepsilon^{\prime}(1-\cos \vartheta) \frac{\partial^{2} \sigma}{\partial \Omega \partial \varepsilon^{\prime}},
$$

where $\vartheta$ denotes the scattering angle. These cross sections are employed for a simple description of the slowing down process of different particles. The transport cross sections for $p \mu(F=0)$ scattering in the liquid and gaseous para- $\mathrm{H}_{2}$ are plotted in Figure 6. 


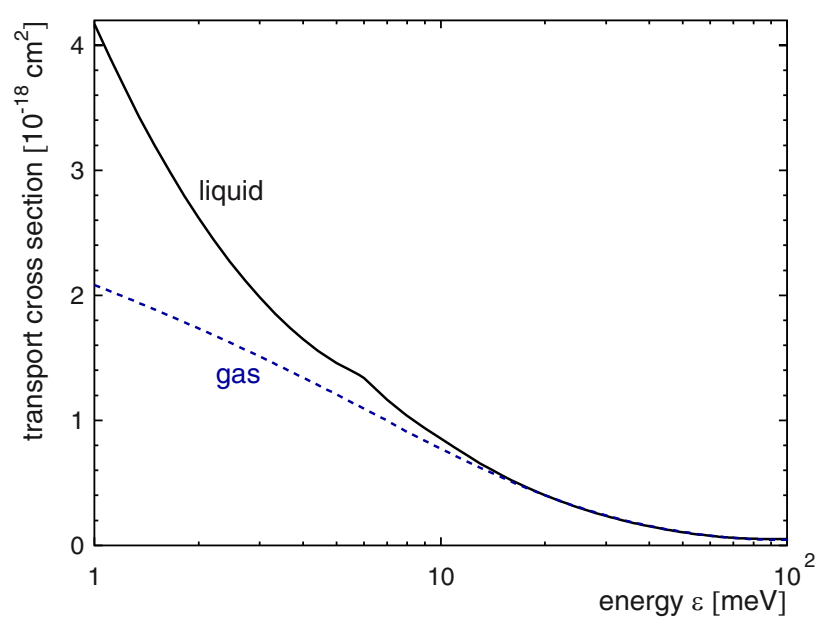

Fig. 6. Transport cross sections for $p \mu(F=0)$ scattering in the $22-\mathrm{K}$ liquid and gaseous para- $\mathrm{H}_{2}$.

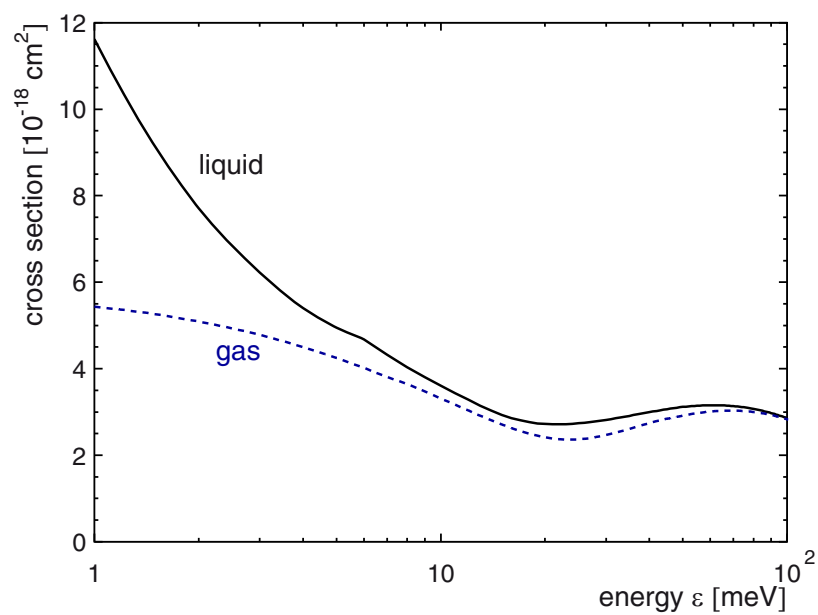

Fig. 7. The same as in Figure 5 for $p \mu(F=1)$.

The cross section for scattering of the $p \mu$ atom in the excited spin state $F=1$ significantly differs from that for the ground spin state $F=0$. The amplitudes of $p \mu(F=0)$ and $p \mu(F=1)$ scattering from a proton have the opposite signs and quite different magnitudes. Moreover, in the case of $p \mu(F=1)$ scattering from the $\mathrm{H}_{2}$ molecule, the ortho-para rotational transitions are allowed due to the proton exchange between the atom and the $\mathrm{H}_{2}$ molecule. A difference of the both cross sections is apparent while comparing Figure 5 with Figure 7 . Other differences between the cross sections are due to the scattering in hydrogenic targets with various populations of the molecular rotational states. The cross sections for the $p \mu(F=1)$ scattering in liquid and gaseous normal hydrogen are presented in Figure 8 as an example. In $n \mathrm{H}_{2}$, a population of the excited rotational state $K=1$ is equal to $75 \%$. As a result, the rotational deexcitation $K=1 \rightarrow 0$ is possible, which significantly changes the cross sections at lowest energies (see Figs. 7 and 8).

Energies of the hyperfine splitting in the muonic hydrogen atoms (182 meV for $p \mu$ and $48.5 \mathrm{meV}$ for $d \mu$ ) are much greater than the Debye energy of about $6 \mathrm{meV}$.

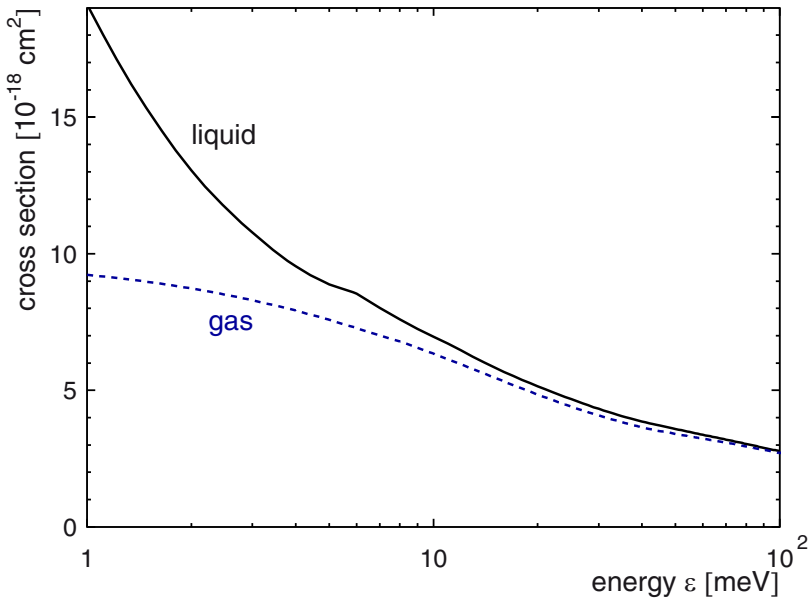

Fig. 8. Total cross sections for $p \mu(F=1)$ scattering in liquid and gaseous $\mathrm{nH}_{2}$.

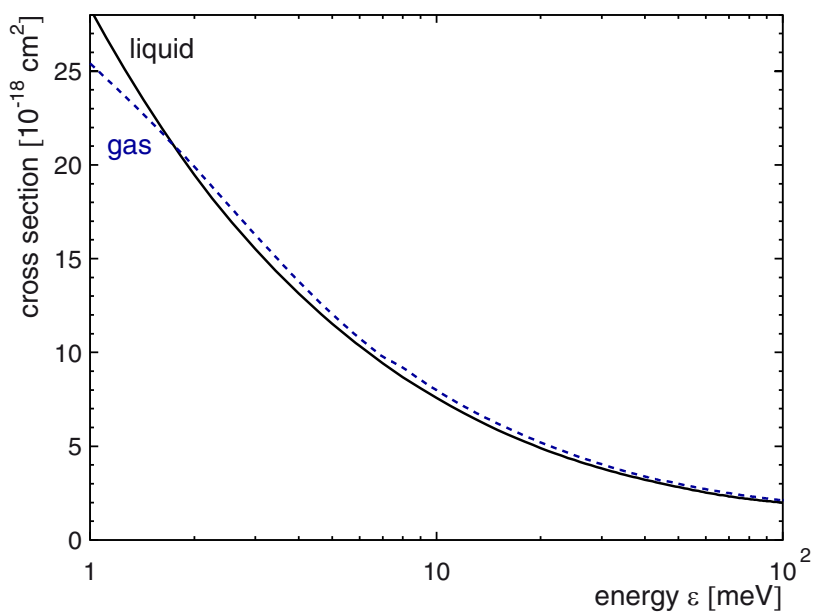

Fig. 9. Total cross sections for the spin flip process $p \mu(F=$ 1) $+\mathrm{H}_{2} \rightarrow p \mu(F=0)+\mathrm{H}_{2}$ in the $22-\mathrm{K}$ liquid and gaseous para- $\mathrm{H}_{2}$.

Therefore, the energy transfer $\omega$ in the spin-flip transitions is relatively large. As a result, the spin-flip cross sections in $a \mu$ scattering from the liquid hydrogens only slightly differ from the corresponding cross sections for hydrogenic gases. The reaction $p \mu(F=1)+\mathrm{H}_{2} \rightarrow p \mu(F=$ $0)+\mathrm{H}_{2}$ in the liquid and gaseous parahydrogen is plotted in Figure 9. An significant difference between the spinflip cross sections is apparent only below $2 \mathrm{meV}$. Let us note that the effective spin-flip reaction in the scattering $p \mu+\mathrm{H}_{2}$ is due to the muon exchange between the protons [29].

Figures 10 and 11 present the total and transport cross sections for scattering of the $d \mu$ atom in the ground spin state $F=1 / 2$ from the liquid and gaseous orthodeuterium at $22 \mathrm{~K}$. The corresponding cross sections for $d \mu$ scattering in the upper spin state $F=3 / 2$ are similar since the mean amplitudes for the scattering $d \mu(F=1 / 2)+d$ and $d \mu(F=$ $3 / 2)+d$ are quite close. The mean amplitude denotes here averaging over the total spin of the system $d \mu+d$. Moreover, the rotational transition $K=1 \rightarrow 0$ in the scat- 


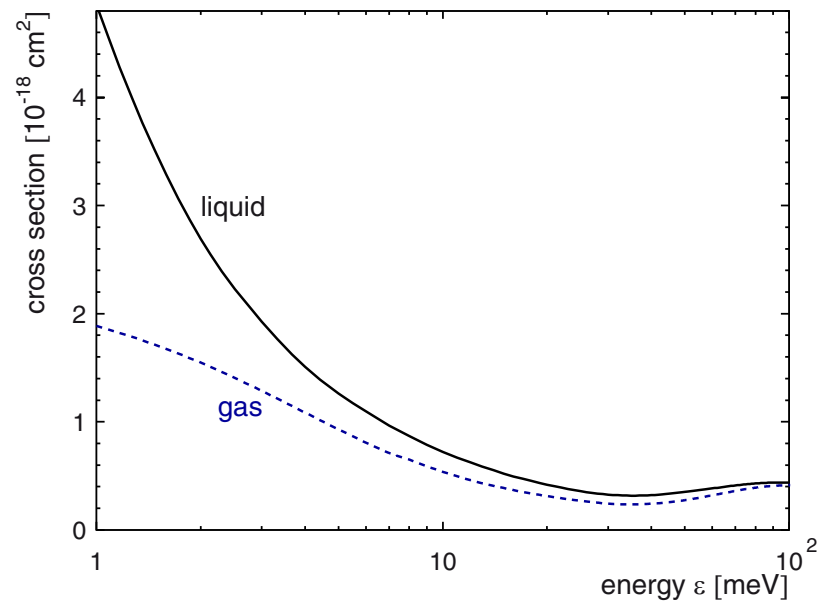

Fig. 10. Total cross sections for $d \mu(F=1 / 2)$ scattering in the $22-\mathrm{K}$ liquid and gaseous ortho- $\mathrm{D}_{2}$.

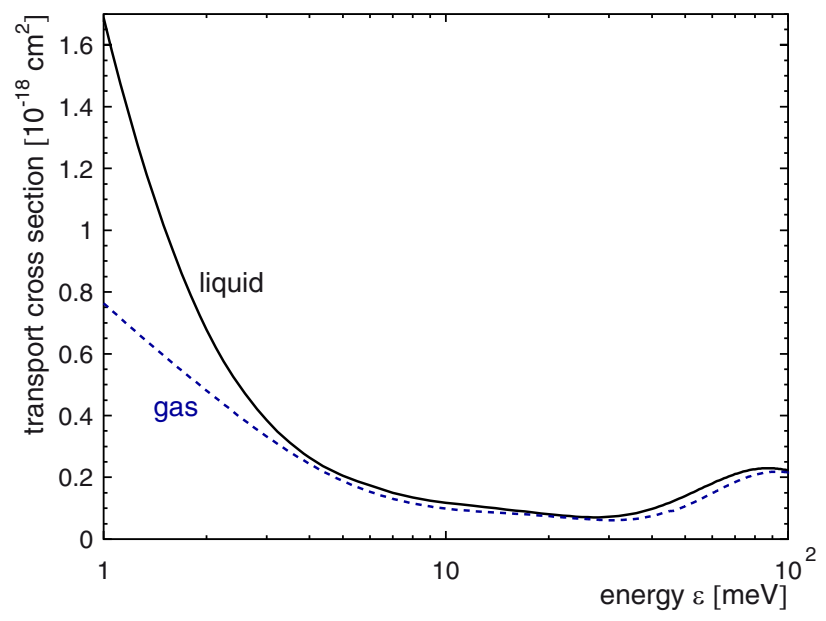

Fig. 11. Transport cross sections for $d \mu(F=1 / 2)$ scattering in the 22-K liquid and gaseous ortho- $\mathrm{D}_{2}$.

tering $d \mu+\mathrm{D}_{2}$ is allowed for the both states $F=1 / 2$ and $3 / 2$. As a result, the cross sections for $d \mu(F=1 / 2)$ and $d \mu(F=3 / 2)$ scattering in $\mathrm{nD}_{2}$, where the population of the rotational ortho-state $K=0$ equals $2 / 3$, are similar. The cross sections of the spin-flip process $d \mu(F=3 / 2)+\mathrm{D}_{2} \rightarrow d \mu(F=1 / 2)+\mathrm{D}_{2}$ in the $22-\mathrm{K}$ liquid and gaseous ortho- $\mathrm{D}_{2}$ are shown in Figure 12. A relative difference between these cross sections is significantly greater $(17 \%$ at $1 \mathrm{meV})$ than in the $p \mu+\mathrm{H}_{2}$ case. This is caused by a smaller hyperfine splitting in the $d \mu$ atom.

The experimental studies of fusion reactions in the muonic molecules $p d \mu$ and $p t \mu$ are often performed in liquid $\mathrm{H}_{2}$ targets with small admixtures $(\lesssim 1 \%)$ of deuterium or tritium $[2-4,12]$. Small amounts of the heavier isotopes do not practically change the density of liquid hydrogen and the sound velocity. In the case of $d \mu$ scattering in hydrogen with a small concentration of deuterium, collisions with the $\mathrm{H}_{2}$ molecules are most frequent. The cross sections for $d \mu$ scattering from the $\mathrm{H}_{2}$ molecules in the 22-K liquid and gaseous H/D mixture are plotted in Figure 13. These cross sections does not depend on

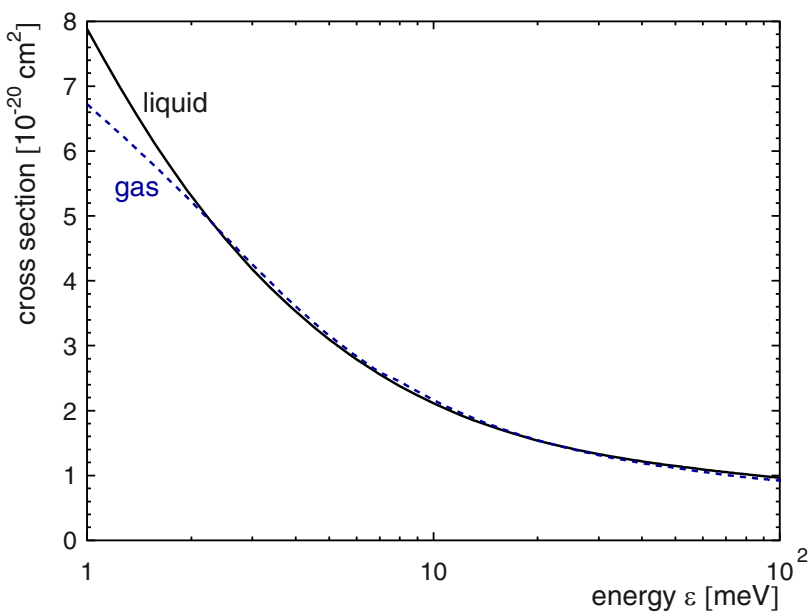

Fig. 12. Total cross sections for the spin flip process $d \mu(F=$ $3 / 2)+\mathrm{D}_{2} \rightarrow d \mu(F=1 / 2)+\mathrm{D}_{2}$ in the $22-\mathrm{K}$ liquid and gaseous ortho- $\mathrm{D}_{2}$.

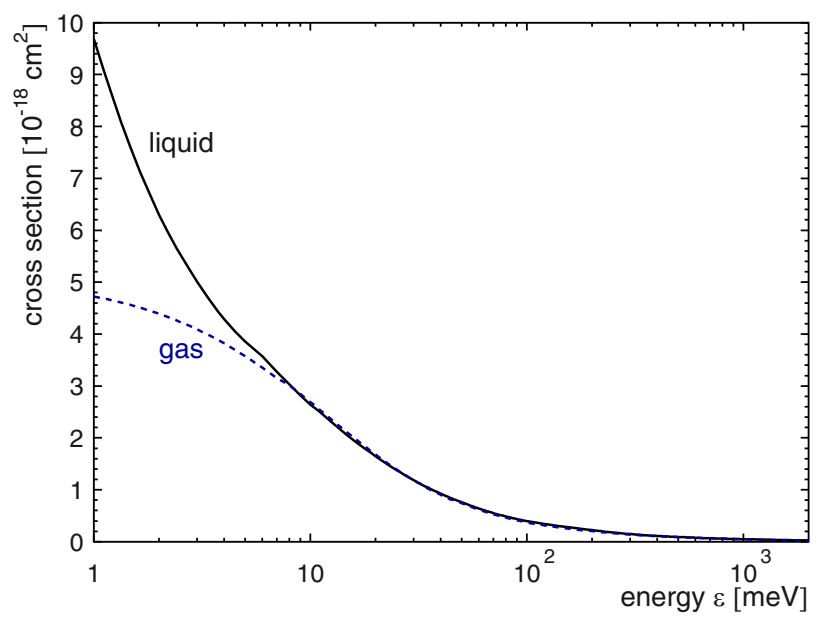

Fig. 13. Total cross sections for $d \mu$ scattering from the $\mathrm{H}_{2}$ molecule in the $22-\mathrm{K}$ liquid and gaseous para- $\mathrm{H}_{2}$.

the spin of $d \mu$. In such H/D mixtures, $d \mu$ collision with the deuterons takes place mostly in the HD molecules, since the concentration of $\mathrm{D}_{2}$ molecules in the equilibrated $\mathrm{H} / \mathrm{D}$ mixture is very small. The total cross sections for $d \mu(F=1 / 2)$ scattering from the HD molecule in liquid and gaseous hydrogen are plotted in Figure 14. This molecule is in the ground rotational state since the asymmetric hydrogenic molecules rapidly deexcite to the state $K=0$ at low temperatures. For calculating the response

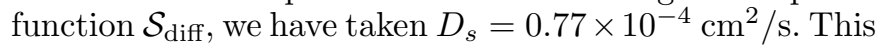
is a value of the diffusion coefficient for a $D_{2}$ molecule which diffuses in liquid $\mathrm{H}_{2}[26]$. However, this is a fair approximation for the molecules HD and HT moving in liquid hydrogen, at small concentrations of deuterium and tritium. The total cross sections for $t \mu$ scattering from the $\mathrm{H}_{2}$ and HT molecules in the liquid and gaseous $\mathrm{H} / \mathrm{T}$ mixture (small tritium concentration) are presented in Figures 15 and 16.

In the kinetics equations for the muon-catalyzed fusion in $\mathrm{H} / \mathrm{D} / \mathrm{T}$ mixtures, the rates of different important 


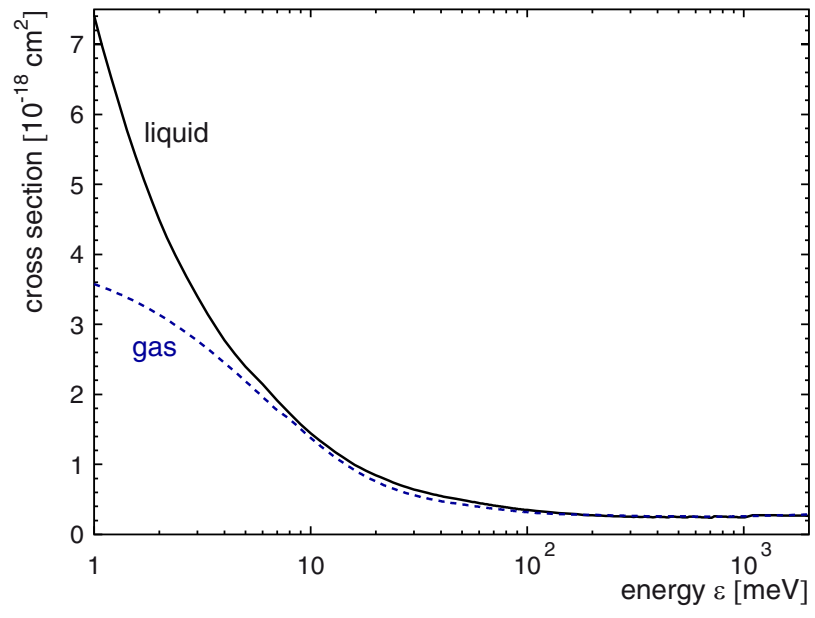

Fig. 14. Total cross sections for $d \mu(F=3 / 2)$ scattering from the HD molecule in the $22-\mathrm{K}$ liquid and gaseous para- $\mathrm{H}_{2}$.

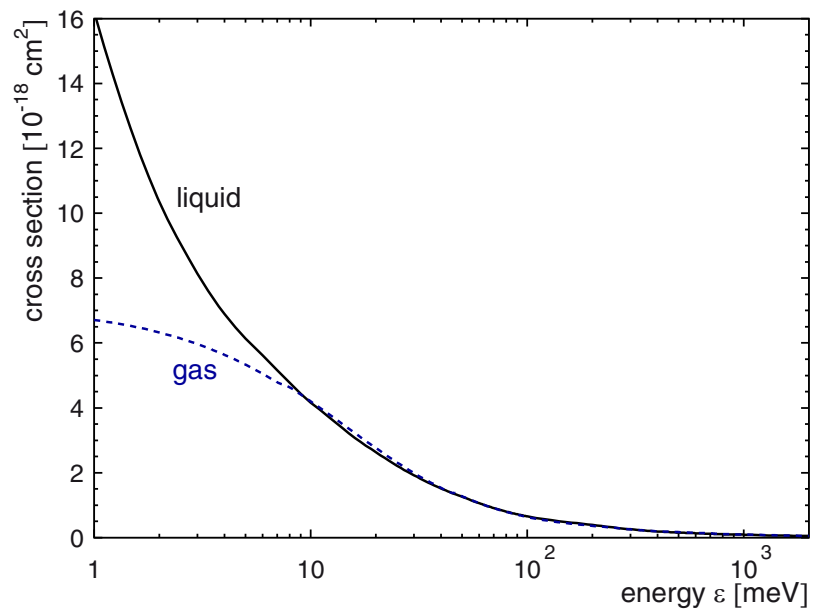

Fig. 15. Total cross sections for $t \mu$ scattering from the $\mathrm{H}_{2}$ molecule in the $22-\mathrm{K}$ liquid and gaseous para- $\mathrm{H}_{2}$.

reactions are employed. In particular, the spin-flip rates are needed for a correct description of the time spectra of fusion products. In Figure 17 we present the calculated spin-flip rates for $d \mu(F=3 / 2)$ scattering from the HD molecule and $t \mu(F=1)$ scattering from the HT molecule in liquid hydrogen. The rates are normalized to the density of $2.12 \times 10^{22}$ mols $/ \mathrm{cm}^{3}$, which is common in low-energy muon physics. This is the number density of liquid hydrogen at $20.4 \mathrm{~K}$ and saturated-vapor pressure.

\section{Conclusions}

The differential cross sections for low-energy scattering of the muonic hydrogen atoms from liquid hydrogens have been estimated in the incoherent approximation. The Van Howe response function and the simple model of liquid hydrogen, which takes the diffusive and vibrational degrees of freedom into account, have been employed. Our calculations show that effects associated with the presence of liquid are very important for kinetic energies below $10 \mathrm{meV}$. Above $100 \mathrm{meV}$, the corresponding cross

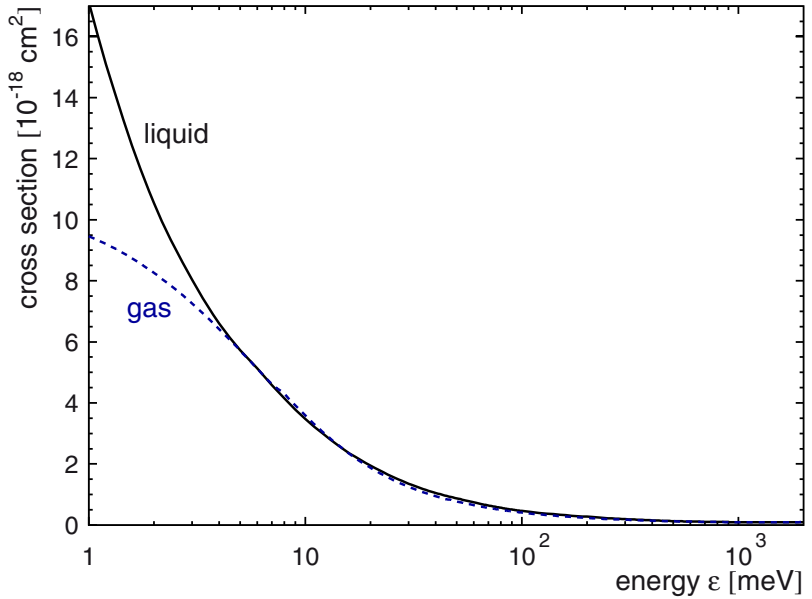

Fig. 16. Total cross sections for $t \mu(F=0)$ scattering from the $\mathrm{HT}$ molecule in the $22-\mathrm{K}$ liquid and gaseous para- $\mathrm{H}_{2}$.

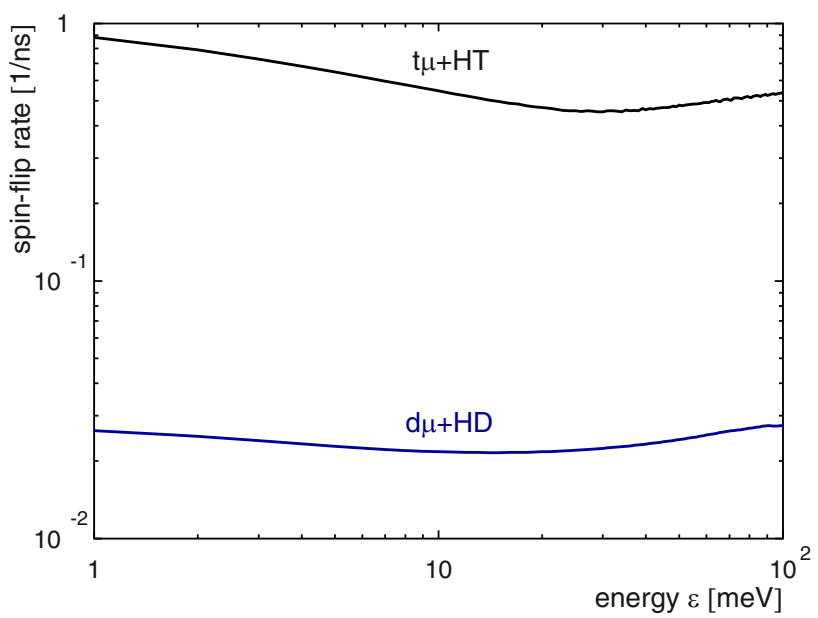

Fig. 17. Total rates of spin deexcitation in the scattering $d \mu(F=3 / 2)+\mathrm{HD}$ and $t \mu(F=1)+\mathrm{HT}$ in the $22-\mathrm{K}$ liquid hydrogen.

sections for scattering in liquid and gaseous hydrogens are practically identical. In the case of spin-flip reactions, an appreciable difference between these cross sections is apparent only below a few $\mathrm{meV}$, which is caused by a relatively high (compared to the Debye energy) energy transfers to the target. This difference $(12 \%$ for the scattering $p \mu(F=1)+\mathrm{H}_{2}$ and $17 \%$ for $d \mu(F=3 / 2)+\mathrm{D}_{2}$ at $1 \mathrm{meV}$ ) is however significant for the spin-dependent processes, before a steady-state condition is reached.

Thermalization of the energetic $(\sim 1 \mathrm{eV}) p \mu$ atoms in a pure liquid hydrogen is fast ( $\lesssim 10 \mathrm{~ns})$ owing to high magnitudes of the scattering cross sections and a large number density of the target molecules. The same conclusion holds for thermalization of the $d \mu$ atoms in a pure liquid deuterium. As a result, the time spectra, such as the spectrum of $d d$-fusion products, are affected by properties of the liquid only at short times ( $\lesssim 10 \mathrm{~ns})$.

Another situation can be observed in the case of liquid hydrogen with small admixtures of deuterium and tritium. The negative muons from a beam are captured 
mostly by the $\mathrm{H}_{2}$ molecules, which leads to formation of the $p \mu$ atoms and subsequent formation of the $d \mu$ or $t \mu$ atoms in the muon exchange reactions $p \mu+d \rightarrow d \mu+p$ or $p \mu+t \rightarrow t \mu+p$. The released energy greatly accelerates the new atoms. They cannot be effectively slowed down to thermal energies in scattering from the abundant $\mathrm{H}_{2}$ molecules because of the deep Ramsauer-Townsend minima in the elastic scattering processes $d \mu+p$ and $t \mu+p$ at laboratory energies of about $5 \mathrm{eV}$ and $10 \mathrm{eV}$ [10], respectively. As a result, scattering from the molecules HD and HT establishes an effective mechanism of deceleration and spin deexcitation of the $d \mu$ and $t \mu$ atoms, due to the presence of the deuteron or triton in the target molecules. Since the concentrations of deuterium and tritium are small, the region of thermal energies is reached at much larger times than in the case of pure liquid $\mathrm{H}_{2}$ and $\mathrm{D}_{2}$. Therefore in such $\mathrm{H} / \mathrm{D} / \mathrm{T}$ mixtures, at large times, one can expect significant effects associated with the dynamics of the hydrogenic liquids.

Helpful discussions with Drs. D.L. Demin and M.P. Faifman are gratefully acknowledged.

\section{Author contribution statement}

Both authors have equally contributed to this paper.

Open Access This is an open access article distributed under the terms of the Creative Commons Attribution License (http://creativecommons.org/licenses/by/4.0), which permits unrestricted use, distribution, and reproduction in any medium, provided the original work is properly cited.

\section{References}

1. J.H. Doede, Phys. Rev. 132, 1782 (1963)

2. E.J. Bleser et al., Phys. Rev. 132, 2679 (1963)
3. C. Petitjean et al., Muon Catal. Fusion 5/6, 199 (1990/91)

4. P. Baumann et al., Phys. Rev. Lett. 70, 3720 (1993)

5. V.E. Markushin, P. Baumann, F.J. Hartmann, Hyperfine Interact. 77, 161 (1993)

6. L. Wolfenstein, in Proceedings of the 1960 Annual International Conference on High Energy Physics at Rochester (Interscience Publishers, Inc., Rochester, N.Y., 1960), p. 533

7. Ya.B. Zeldovich, S.S. Gershtein, Usp. Fiz. Nauk. 71, 581 (1960) [Sov. Phys. Usp. 3, 593 (1961)]

8. M. Bubak, M.P. Faifman, Preprint JINR E4-87-464 (Joint Institute for Nuclear Research, Dubna, Russia, 1987)

9. L. Bracci et al., Muon Catal. Fusion 4, 247 (1989)

10. C. Chiccoli et al., Muon Catal. Fusion 7, 87 (1992)

11. A. Adamczak, Phys. Rev. A 76, 052512 (2007)

12. L.N. Bogdanova et al., Phys. El. Part. At. Nucl. Lett. 9, $605(2012)$

13. L.N. Bogdanova, Phys. Atom. Nucl. 76, 376 (2013)

14. L. Van Hove, Phys. Rev. 95, 249 (1954)

15. A. Adamczak, Phys. Rev. A 74, 042718 (2006)

16. F.J. Bermejo et al., Phys. Rev. B 47, 15097 (1993)

17. F.J. Bermejo et al., Phys. Rev. B 60, 15154 (1999)

18. F.J. Bermejo et al., Phys. Rev. Lett. 84, 5359 (2000)

19. M. Mukherjee, F.J. Bermejo, S.M. Bennington, B. Fåk, Phys. Rev. B 57, R11 031 (1998)

20. F.J. Mompeán, M. Garcia-Hernández, B. Fåk, Phys. Rev. B 56, 11604 (1997)

21. J.R. Granada, V.H. Gillette, Physica B 348, 6 (2004)

22. E. Guarini et al., Phys. Rev. B 92, 104303 (2015)

23. E. Guarini et al., Phys. Rev. B 93, 224302 (2016)

24. S.W. Lovesey, Theory of neutron scattering from condensed matter (Clarendon Press, Oxford, 1984)

25. P.A. Egelstaff, P. Schofield, Nucl. Sci. Eng. 12, 260 (1962)

26. P.C. Souers, Hydrogen properties for fusion energy (University of California Press, Berkeley, 1986)

27. P. Résibois, M. de Leener, Classical kinetic theory of fluids (Wiley, New York, 1977)

28. M. Zoppi, D. Colognesi, M. Celli, Eur. Phys. J. B 23, 171 (2001)

29. S.S. Gershtein, Zh. Eksp. Teor. Fiz. 34, 463 (1958) [Sov. Phys. JETP 7, 318 (1958)] 